son, during the time she was under my care, has been received, and I now cheerfully comply therewith.

On the day following her delivery and upon which you were taken ill, I was requested to visit her merely for the purpose of relieving her bladder.

After the lapse of seven days, at about I:30 o'clock on the following Thursday morning, the 20 th, I was called the second time to see her, because of a violent chill with which she had been attacked soon after midnight. Her temperature at this visit was $106^{\circ} \mathrm{F}$., but in a very short time, during a copious perspiration, declined to about IoO or IOI.

Agreeable to the most approved treatment in these cases, regarding her case as a typical one of puerperal pyæmia, resort was now had to intra-uterine injections of carbolized warm water and to the internal administration of quinine, ten grain doses, at intervals of four hours. For a time she seemed greatly improved and apparently approaching convalescence. Suddenly, without any premonition, she was taken with another chill, which was accompanied with the same phenomena already referred to. And so chill after chill followed each other in irregular succession, each attended with a rapid rise and decline of the temperature with profuse sweating. The pulse after the third rigor had attained an acceleration of 140 , and evidences of great exhaustion were now beginning to be manifest.

Upon making inquiry of the husband I learned of the difficulty you had in extracting the placenta. I concluded that you must have had one of those rare cases of true adherent placenta, in which it is utterly impossible to effect its entire separation, the union being so firm. I then remarked to Mr. Ellison that the cduse of this adhesion must certainly have been due to the injury she sustained in the accident which befell her a few months prior, for reasons which you will readily understand, and I believe this accident rendered her general system illy prepared to resist the influence which the difficult extraction of the placenta would exert in consequence of the septic dangers it would occasion.

Very truly yours,

H. V. SWERINGEN.

\section{ADDRESS IN OBSTETRICS AND DISEASES OF WOMEN.}

BY THAD. A. REAMY, M.D., OF CINCINNATI, OHIO, CHAIRMAN OF THE SECTION.

[Read in General Session of the American Medical Association, May, 1884.]

Fellow Members :-Custom, as well as law, prescribes as one of the duties of the Chairman of a Section that he present in his annual address a digest of the progress made in that special department during the past year.

Compliance with the letter of this law is now unnecessary, and would, therefore, be unprofitable.
Medical journals, general and special, both home and foreign, conducted with great ability and exhibiting much energy and zeal in collecting and publishing the latest advances in all departments, are promptly placed by rapid mail facilities upon the table of every reading member of the profession. Moreover, the editorial criticisms of new doctrines, discoveries, methods or operations, are generally from the pens of those who, by education and special training, have superior abilities for such work. It is, therefore, deemed appropriate, on the present occasion, to refer you for an "Annual Report on Obstetrics and Diseases of Women " to these more fertile and instructive sources.

Craving your indulgence, I beg to offer, in lieu of such an address,

NOTES OF TWO HUNDRED AND THIRTY-ONE CASES OF OPERATION FOR LACERATION OF THE CERVIX UTERI.

The date of my first operation for lacerated cervix, "Emmet's operation," was February 28, I 874. I have now made the operation upon $23^{I}$ patients. Not a single death has occurred. In six cases the operation was followed by perimetritis, parametritis or peritonitis. In but three of these cases, however, were these symptoms sufficiently severe to cause material delay in complete recovery. In one case, included in these three, there was perimetritis, paramatritis, and general peritonitis. The patient was confined to her bed for three months. During the attack (acute stage) the peritoneal cavity was aspirated twice. On one occasion seventeen ounces of serum were removed. This woman finally recovered her health perfectly. Considerable fixedness of the uterus remained for a long time; but now, at the expiration of two and a half years, uterine mobility is about normal, menstruation normal and painless, whereas prior to the operation she suffered both from menorrhagia and dysmenorrhœe. She was the mother of two children, the youngest being 4 years old at the date of operation. No abortions had occurred. Sterility continues. The above case was one of bilateral laceration, extending on each side to the vaginal junction, the torn lips being widely separated and eroded. I attribute the inflammatory complications following the operation in this case to the undue degree to which the uterus was dragged down in order to give unobstructed access. On this point I shall have something further to say before the close of this paper.

Complete recovery occurred in the other two cases, but convalescence was slow.

Of these $23 \mathrm{I}$ cases, in $\mathrm{I} 70$ the laceration was bilateral, in $3^{8}$ unilateral. Of these latter, 23 were on the left side and 5 on the right. Sixteen cases were stellate, in two of which there were four distinct lacerations. In five cases there was laceration of the posterior lip only, in two, of the anterior lip only. In 80 cases the laceration was extensive; in ${ }_{5} 5$ of these 80 extending to the cervico-vaginal junction on both sides. In 22 of the bilateral cases the laceration extended to the cervico-vaginal junction on one side only. In three cases the rent extended to the internal os. In one of these, the vaginal wall was 
likewise extensively lacerated; the peritoneal cavity had probably been opened, followed by protracted cellulitis. This patient remained a helpless invalid during the three years intervening between the accident and the date of my operation. On one side it required 12 sutures to close the cervical and vaginal rent. Perfect success followed the operation, the patient being restored to robust health within six months.

In 167 cases there was perineal laceration to an extent that left deformity, either at the vaginal orifice or more externally. In ${ }_{5} 5$ cases the anal sphincter was damaged. In 7 cases the recto-vaginal septum was opened up. In 26 cases I operated upon the cervix and perinæum at the same sitting. In 5 cases of this series I curetted the uterus with my blunt wire curette at the same sitting, this of course, being the primary of the three procedures. In neither of these five cases did a single untoward symptom arise. Results were perfect.

In $5^{\circ}$ cases I operated upon the perinæum after recovery from the cervical operation. Each of the cases above mentioned in which the operation involved the recto-vaginal septum is included in this series of fifty subsequent operations.

It is perhaps as well to state here that I never hesitate to curette the uterus in a case demanding it, at the time of operating upon the cervix. Nor do I hesitate to use the curette in a case requiring it immediately preceding a perineorrhaphy, pretty extensive clinịcal experience having convinced me that neither of these operations adds materially to the danger of curetting. Indeed, I am inclined to the opinion that in certain cases where the conjoined conditions demanding the two operations exist, the depletion of the cervix in trachelorrhaphy presents an element of safety against inflammatory processes which may follow curetting. Three violent cases of traumatic peritonitis have occurred within my personal experience from the use of the curette, unconnected with other operations. In not a single case of trachelorrhaphy performed by me did secondary hæmorrhage orcur.

In every case where hæmorrhage was troublesome during the operation, it was readily controlled by the stream of hot water which it is my custom to have flow upon the field of the operation during its progress, with but three exceptions; in which it being necessary to denude the torn surfaces deeply, twigs of the circular branch of the uterine artery being cut, compression with torsion forceps was invoked. Contrary to most operators I have not, since my fourth operation, employed a tourniquet, ligature, or any similar method to control hæmorrhage during the operation. As early as 1875 , in my clinical lectures delivered in the amphitheater of the Good Samaritan Hospital, and repeated each year since, I emphasized the importance of allowing, in most cases, free bleeding from the denuded surfaces. Firstly $(a)$ because it softens the tissues so as to allow more perfect coaptation of the lips to be united; and $(b)$ because of this softening, which is immediate, the tissues are easily penetrated by the needies in carrying the sutures. Secondly, and more important, this depletion not only greatly promotes the return of the indurated cervical tissue to its normal condition, but is an immense factor in again starting the processes of involution of the uterus which had been arrested by the laceration and its consequences. The restoration of form in closing the divided lips of a torn cervix, especially where the deformity is great, is important. The removal of such pathological conditions as may thrcaten the development of cancer, and no one can deny that this is in a certain proportion of instances within the scope of the operation, may be even more important ; but the influence of trachelorrhaphy, properly done, upon subinvolution cannot be over-estimated. Thorough denudation, cutting out all cicatricial tissue, and aliowing free depletion, embrace the essential points in the operation so far as its influence upon involution is concerned.

So powerful is the influence of hot water in controlling hæmorrhage from small vessels, when it can be poured directly upon the bleeding surface, that I have frequently found it expedient to withhold this agent for a short time after the denudation is completed before introducing the sutures, in order that sufficient bleeding may occur to accomplish the ends. above indicated.

The character of the labor which had resulted in laceration could not be determined with any degree of certainty, except in comparatively few instances, since information upon this point could be obtained only from the patients themselves. In 40 cases delivery was accomplished by the forceps; in 2 by craniotomy ; in 2 by turning. Two of the cases where rupture involved the recto-vaginal septum, were forceps deliveries.

It is my belief that early rupture of the membranes, and attempts at forcible dilatation of the cervix by the finger or fingers of the accoucheur or midwife, and the improper use of ergot, are far more fruitful sources of laceration to the cervix than the obstetric forceps.

In the city of Cincinnati 70 per centum of all deliveries are attended by midwives, who, with very few exceptions, are ignorant and unskilful. The sins above enumerated lie closely at their doors. But it cannot be denied that these errors of practice are sometimes perpetrated by well educated physicians. Unquestionably, many cases of laceration occur unavoidably, no matter what care and skill may characterize the accoucheur's services. It should be the duty, therefore, of the gynæcologist in all cases where patients are referred or otherwise come into his hands for operation, to protect the physician who may have been present at the confinement against censure, which may be unjust. All the more because the laity, when informed that a laceration of either cervix or perinæum has occurred, at once assume a want of skill or care in the obstetric service. The emplatic statement of the unavoidableness of these accidents should by the gynæcologist be voluntary. So great is the danger that professional reputation may unjustly suffer from this source of censure, that in an editorial in a recent issue of one of the ablest medical journals of the country, this was used as an argument against the adoption of Emmet's operation except in extreme cases. 
Mr. Jonathan Hutchinson points out that in many cases the first stage of cancer is that of inflammation; that "all inflammations are infective." "Inflammatory processes may pass by almost imperceptible gradation into malignancy." The acceptance and appreciation of the above facts would lead in many cases to the more vigorous treatment of localized inflammations; more especially when situated in structures known from clinical experience to be specially prone to cancer, as the uterine cervix, mammæ, etc. These considerations alone, even if they were not supported by others, urge with great force upon our attention the importance of Emmet's operation. It is the concurrent testimony of almost every distinguished American gynæcologist who has spoken on the subject, with many abroad, including Briesky and Schroeder, that the fretting of exposed tissue consequent upon ununited laceration of the cervix is a prolific source of danger in developing cancer.

Emmet, Thomas, Goodell, Mundé and others have cited cases verifying this danger. In my own practice quite a number of cases of cancer of the cervix have been observed where the neoplasm developed either in the cicatricial tissue resulting from laceration or in the apex of the cleft.

In my opinion, the influence of the accident under discussion in producing cancer has never been fully apprehended by any one. Emmet, the distinguished author of the operation under consideration, at first spoke out unequivocally. Now, however, he alleges that he does not make trachelorrhaphy once where formerly he did it ten times; and it is easy to read between the lines of what he has recently written, that he does not now believe the evils arising from laceration not operated on, nearly so universal as he formerly believed them to be. He seems greatly to deplore the extent to which the operation is being done, and as I think, without just cause.

In further considering the relations between this injury and Emmet's operation to cancer, it may not be amiss to call attention to the fact so thoroughly recognized by clinicians, that cancer of the cervix is found almost exclusively in women who have either given birth to one or more children or been the subjects of abortion. So far as the cervix is concerned, cancer is a disease of childbearing women, not of virgins. In more than 300 cases of cancer of the cervix of which I have notes but one occurred in a virgin, and but ten in married women in whose cases I could obtain no evidence of abortion or childbearing. Can any one deny that such facts point strongly to the local origin of cancer in these cases at least? And can it be doubted that injuries inflicted upon the cervix during parturition have a direct or remote connection with this origin? Would it not be wise to repair these injuries when they are appreciable by whatever method it may be done most speedily and certainly? It is claimed that Emmet's operation should be limited to cases where ectropion has occurred, or where a cicatricial plug imprisons or presses upon branches of sentient nerves, causing painful reflex symptoms. I have heard it repeatedly stated that no matter how extensive the laceration, the operation is not warranted unless these symptoms are present. These are in my judgment errors. Any laceration which has healed without its surfaces being in contact must have healed without complete union, although its extent may have been much lessened by granulation. But in all such cases there is more or less cicatricial tissue in the field of repair. (This does not apply to the many cases where anterior and posterior laceration occurs, and the parts not being separated complete union has occurred without the aid of the surgeon). Cicatricial tissue in such locality, even should it not produce reflex symptoms, should be removed thoroughly and the parts closed so as to obtain union by first intention, in which cicatricial tissue is never found. If the rent be small, then the operation is small, especially if it be done before chronic inflammation of the cervix has occurred, as a result. The denudation need not be extensive or deep. In many cases an anæsthetic need not be administered, as the parts are not very sensitive. The uterus need not be drawn down, so there can be but little danger of cellulitis or peritonitis resulting. Why not do the operation in these slighter cases, then, as the simplest and safest method of cure? thus avoiding results which might arise in the future and prove more serious?

It is impossibie for me to give exact data as to the influence of the operation on sterility, as many of these cases soon after recovery, passed out of my knowledge. And as the last 50 cases were operated on within the past thirteen months, sufficient time has not elapsed to test the question. I know, however, of I 5 cases where conception has occurred, and delivery took place at term. I have no doubt that as many more have proven fruitful. Of these I attended in labor 6 , one of these within the past month. In no case did relaceration occur, labor being normal except in two cases. In these dilatation of the cervix was slow, but finally complete, in neither case was it protracted. I have learned from medical gentlemen who attended several other of my cases that labor was normal. I believe that the operation properly done, favors fertility, and often cures sterility.

In but 2 of my cases did union fail. In one of these I foolishly used catgut ligature. In the other failure was due to the sutures not being sufficiently tightened. In each case subsequent operation was successful.

I may have made the operation in cases not demanding it, but from my point of view, I do not think so. In 8 or ro cases reflex nervous symptoms, which I hoped to banish by the operation, remained, but in most cases the good effects were obvious; in some cases the cures were almost marvellous.

My method of operating, after much thought, and some experience I think worthy of consideration, as it must, as I believe, have some advantages over methods generally adopted.

I. I use nothing to draw the uterus down with but the single vulsella, with which I seize but one lip, the one to be denuded first, at a time.

2. I draw the uterus down as little as possible. This caution should be the more scrupulously observed, if any cellulitis remains about the base of the broad ligaments or elsewhere. 
3. I outline the denudation with a sharp knife, and then cut the tissue included in the line with a sharp scissors. This prevents the rolling of tissue at the borders.

4. Allow bleeding freely or not, as the condition of the tissue of the cervix and the involution or subinvolution of the uterus may require.

5. Use a nearly half-circle needle with very sharp point, armed with Chinese silk.

This shaped needle can be drawn through the second lip and withdrawn very much more easily than a straight needle, or one curved only near the point. This advantage is all the more apparent when the uterus is not drawn down, as then the vaginal wall makes the withdrawal of the usual shaped needle difficult. I employ a plain needle-holder without any catch or slide, but strong.

Silk is preferred to wire because it can be tied much more quickly, and the tension more easily adjusted. Then there are no ends to jag the vaginal walls.

Another great advantage of the silk over the wire is in the fact that the sutures may be left in ${ }^{5}$ to 30 days, without danger of cutting out-quite an important matter in a case when trachelorrhaphy and perineorrhaphy are done at the same sitting. The perinæum may perfectly heal and be strong before removing cervical sutures. My custom is to allow the ends of the sutures to remain sufficiently long to reach nearly to the vaginal opening. This facilitates their removal.

6 . Wash out the cervical canal at the close of the operation with a recurrent flow syringe to remove any blood that may have found its way there during closure of the sutures.

Have nurse wash out the vagina with warm carbolized water, within an hour after the operation is completed. The vagina is not syringed again until the sixth day, then daily until the patient is dismissed.

Cleanliness is the only antisepsis employed.

Authorities have been but little quoted or referred to, as it was my purpose in this paper simply to give notes of my own cases.

Nore.-According to my observations, about one out of every nine women suffers laceration in first confinement.

ESSAY ON DESPERATE SURGERY IN ITS RELATIONS TO WOMEN; THE PROPER PLACE

\section{FOR IT; WHO SHOULD, AND WHO SHOULD NOT ATTEMPT IT.}

BY R. S. SUTTON, M.D., PITTSBURGH, PA.

[Read in the Section on Obstetris.s and Gynæcology of the American Medical Association, May, 1884.]

Mr. President and Gentlemen :

By desperate surgery I mean operations that endanger the life of the patient.

As they relate to women, such operations are largely intra-abdominal.
That the opening of the cavity of the peritonæum is desperately dangerous, will be denied alone by persons ignorant of American statistics tabulating the results of this kind of surgical interference.

That this subject is worthy of intelligent and intense investigation, is claimed by all who honor the profession of medicine, desire to ameliorate the condition of suffering humanity, and wish to prolong life.

The presentation of a tabulated statement of the intra-abdominal surgical operations performed more or less frequently, will serve to bring the subject clearly before our minds. These are:

r, Normal ovariotomy, Battey's operation.

2, Removal of the ovaries and tubes, Hegar-Tait operation.

3 , Ovariotomy for cystic ovaries, McDowel's operation.

4, Fnucleation per vaginam of the entire uterus, Langenbeck's operation.

5, Supra-vaginal hysterectomy, Clay's operation.

6, Enterotomy, Ramdohr's operation.

7, Gastrotomy, Sedilott's operation.

8 , Resection of the pylorus, Billroth's operation.

9, Removal of the gall bladder, Langenbeck's operation.

ro, Cholecystotomy, Marion Sim's operation.

I I, Hepatotomy for hydatids, Lawson Tait's operation.

I 2 , Nephrectotomy, Simon's operation.

I3, Nephrotomy.

I 4, Radical operations for hernia.

15, Laparotomy for pelvic abscess, Lawson Tait's operation.

16, Laparotomy for splenic abscess.

I 7 , Laparotomy for hepatic abscess.

I 8, Laparotomy for chronic peritonitis, Lawson Tait's operation.

I9, Laparotomy for acute peritonitis, Lawson Tait's operation.

20, Laparotomy for hydatids of peritonæum.

2I, Laparotomy for extra uterine fotation, Tait's method.

22, Supra-vaginal hysterectomy in pregnancy, Porro's operation.

In this enumeration there are twenty-two operations within the peritoneal sac, and it is probable that this number will be increased in the near future.

These, with laparotomy for diagnostic purposes, are included in my definition of desperate surgery in relation to women.

May I proceed a step further and characterize this class of surgical operations as a special field of work?

May I affirm that abdominal surgery constitutes a great and growing specialty?

May I predict the gradual limitation of that now ascendant specialty, gynæcology, caused by the special surgeon usurping its abdominal prerogatives?

Is not this the form we see outlined in the shadow of coming events?

It is an admitted fact that when certain organs within the cavity of the peritonæum become diseased, the surgeon's knife is the only available medicament.

Truly the successful gynæcologist is the one that handles the implements of the surgeon skilfully, and 